\title{
Non-steady-state photoelectromotive force in AIN crystal
}

\author{
M. Bryushinin, V. Kulikov, E. Mokhov, S. Nagalyuk, I. Sokolov \\ A.F. Ioffe Physical Technical Institute, 194021, Polytekhnicheskaya 26, St. Petersburg, Russia, \\ i.a.sokolov@mail.ioffe.ru
}

\begin{abstract}
We report the experimental investigation of the non-steady-state photoelectromotive force in aluminium nitride crystal. The sample is illuminated by an oscillating interference pattern formed by two coherent light beams and the alternating current is detected as a response of the material. The experiments are performed for two geometries, where arising photocurrent is parallel or perpendicular to the optical axis of the crystal. Dependencies of the signal amplitude versus light intensity, temporal and spatial frequencies are measured. The photoelectric parameters of the material are estimated for the light wavelength $\lambda=532 \mathrm{~nm}$.
\end{abstract}

Key words: aluminium nitride, non-steady-state photoelectromotive force.

\section{Introduction}

Aluminium nitride (AIN) stands out against other III-V compound semiconductors for its large band gap energy of $6.2 \mathrm{eV}$. This ensures its utilization in detection of visible and UV light, optical modulators, LEDs and lasers [1]. Chemical stability, high mechanical hardness (7 in Mohs scale) [2], high melting temperature, sufficient thermal conductivity $\left(3.19 \mathrm{Wcm}^{-1} \mathrm{~K}^{-1}\right)$ [3] and electron mobility (300 $\left.\mathrm{cm}^{2} \mathrm{~V}^{-1} \mathrm{~s}^{-1}\right)$ [4] also promote its application in electronic devices operating in difficult environments.

AlN crystal has wurzite hexagonal structure with unit-cell dimensions $\mathrm{a}=3.111 \AA$ and $\mathrm{c}=4.980 \AA$ [2]. Crystal lattice belongs to $6 \mathrm{~mm}$ point symmetry group, which allows presence of the electrooptic effect in particular. The first, to our knowledge, observation of the photorefractive effect in AlN, i.e. photoinduced change of the refractive index by means of electrooptic effect, was reported in Ref. 5. The photorefractive holograms recorded in this material demonstrated behavior and characteristics similar to those of "classical" photorefractive crystals $\left(\mathrm{Bi}_{12} \mathrm{SiO}_{20}, \mathrm{LiNbO}_{3}\right)$.

The photorefractive effect includes the stage of space charge formation, which occurs via migration of charge carriers, i.e. electric current. This current is the basis of the non-steady-state photoelectromotive force (photo-EMF) effect, which reveals itself as an alternating electric signal arising in the sample illuminated by an oscillating interference pattern [ 6 , 7]. Since the current is resulted from the periodic relative shifts of the photoconductivity and space charge gratings, the technique based on this effect allows determination of the number of photoelectric parameters (conductivity, carrier sign, lifetime, diffusion length, and drift mobility) and can be applied for the investigation of both noncentrosymmetric and centrosymmetric media. In this paper we study the non-steady-state photo-EMF effect in AlN bulk crystal and apply this technique for determination the photoelectric properties of the material.

\section{Experimental Setup}

Experimental setup for investigation of the nonsteady-state photo-EMF in semiconductors. EOM is the electrooptic modulator, BS is the beam splitter, $\mathrm{M}$ are the mirrors, $\mathrm{A}$ are the amplifiers. Axis [0001] is perpendicular to the figure plane for $\perp$-geometry.

The experimental arrangement used for the investigation of the non-steady-state photo-EMF in AlN crystal is shown in Fig. 1. The second harmonic of Nd:YAG laser $\left(\lambda=532 \mathrm{~nm}, \mathrm{P}_{\text {out }}=20 \mathrm{~mW}\right)$ laser was split into two beams forming the interference pattern with spatial frequency $\mathrm{K}$ and contrast $\mathrm{m}=$ 0.48 on the sample's surface. The signal beam was phase modulated with frequency $\omega$ and amplitude $\delta$ $=0.62$ by the electrooptic modulator ML-102A. The polarization plane of the light was perpendicular to the incidence plane. The beams were expanded in order to illuminate the whole interelectrode spacing. Two sample orientations were studied with the parallel orientation of the grating vector $\mathbf{K}$ and the 


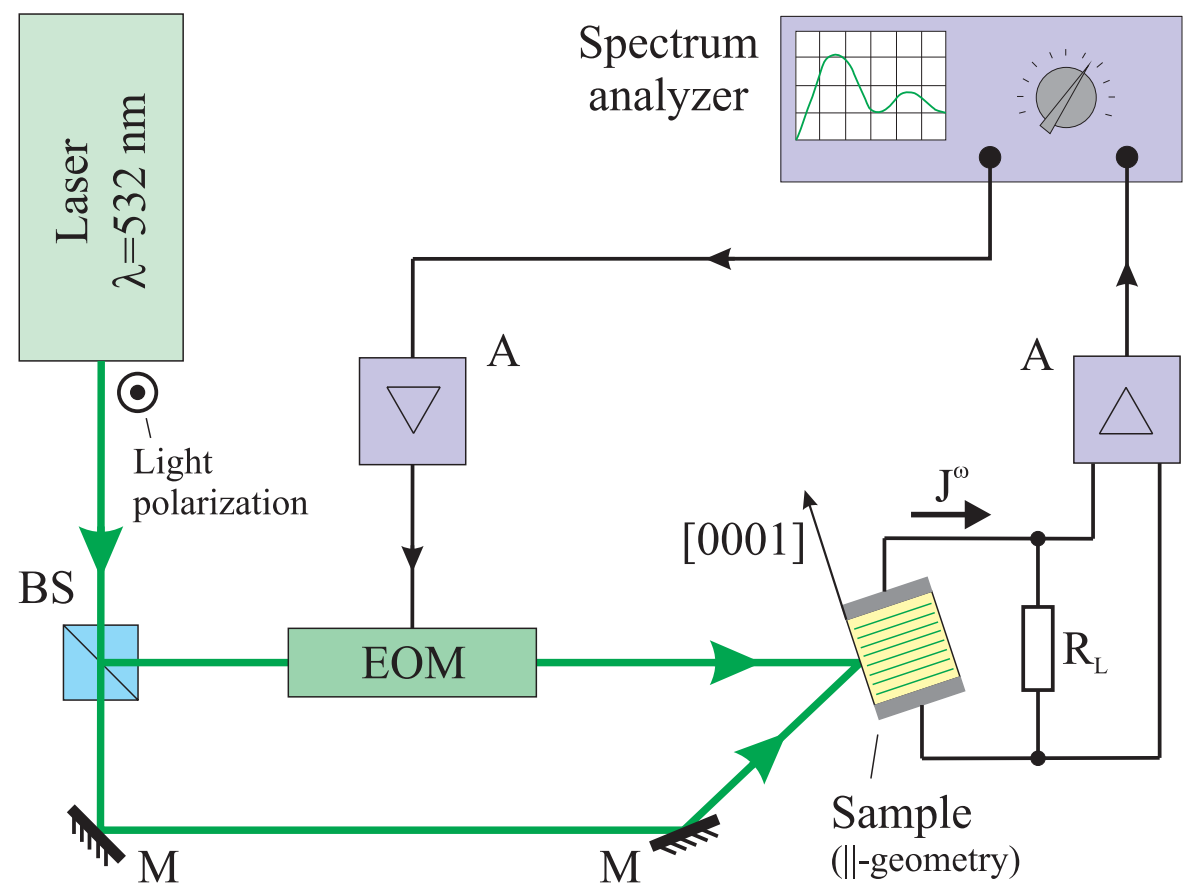

Fig. 1. Experimental setup for investigation of the non-steady-state photo-EMF in semiconductors. EOM is the electrooptic modulator, BS is the beam splitter,M are the mirrors, and A are the amplifiers. The axis [0001] is perpendicular to the figure plane for transverse ( ) geometry.

optical $c$-axis [0001] in the former case ("longitudinal" ||-geometry), and with the perpendicular orientation in the latter one ("transversal" $\perp$-geometry). The photocurrent arising in the sample generated the corresponding voltage on the load resistor $\mathrm{R}_{\mathrm{L}}=10 \mathrm{M} \Omega$. The voltage was amplified and measured using spectrum analyzer SK4-56 (detection bandwidth $\Delta \mathrm{f}=3 \mathrm{~Hz}$ ) or lock-in nanovoltmeter Unipan-232B (with integration time $\tau_{\text {int }}=1-100 \mathrm{~s}$ ). The input resistance of the preamplifier was of $100 \mathrm{M} \Omega$. The sum of the sample and input capacitances was of $3 \mathrm{pF}$. The corresponding correction of the frequency transfer function was performed during calculation of the current amplitude.

The crystal was grown by the physical vapor transport (PVT) technique [8] and has pale yellow color. The characteristic dimensions of the sample were $6.0 \times 3.8 \times 3.0 \mathrm{~mm}^{3}$. The front and back surfaces $\left(3.8 \times 3.0 \mathrm{~mm}^{2}\right)$ were polished. The silver paste electrodes were painted on the lateral surfaces $\left(6.0 \times 3.0 \mathrm{~mm}^{2}\right)$ for $\|$-geometry and $6.0 \times 3.8 \mathrm{~mm}^{2}$ for $\perp$-geometry). The static dielectric constant of the material is of $\varepsilon_{\Pi}=9.14-9.32$ and $\varepsilon_{\perp}=7.76$ $[9,10]$.

\section{Experimental Results}

The presence of the non-steady-state photo-EMF effect in AIN bulk crystal is the first result which should be pointed out. The amplitude of the detected signal is of order of $0.1-1 \mathrm{pA}$ which is noticeably lower than that in the model objects -- sillenite crystals $\mathrm{Bi}_{12} \mathrm{Si}(\mathrm{Ti}, \mathrm{Ge}) \mathrm{O}_{20}$, where it reaches $1 \mathrm{nA}$ [6]. Nevertheless this amplitude is quite enough for consistent detection of the signal with signal-tonoise ratio of $1-10$. The phase of the detected signal indicates that electrons prevail in the process of photo-EMF excitation at low and moderate spatial frequencies of the interference pattern.

We have measured the frequency transfer functions of the non-steady-state photo-EMF signal (Fig. 2) in the "longitudinal" $(\|)$ and "transversal" $(\perp)$ geometries of the experiments, i.e. for the cases when direction of the space charge field $\mathbf{E}_{\mathrm{sc}}$, grating vector $\mathbf{K}$, and photocurrent $\mathbf{J}^{\omega}$ is parallel or perpendicular to the $c$-axis. The signal demonstrates typical behavior, namely, there is a linear growth of the amplitude for low frequencies of the phase modulation $\omega<\omega_{1}$, frequency independent region for higher frequencies $\omega_{1}<\omega<\omega_{2}$, and decay for frequencies $\omega>\omega_{2}$. These regions are separated by the so-called cut-off frequencies $\omega_{1} / 2 \pi=140-700 \mathrm{~Hz}$ and $\omega_{2} / 2 \pi=13-30$ $\mathrm{kHz}$. These dependencies are well described by the following expression which is equivalent to that obtained earlier for the simplest model of the semiconductor crystal with one type of partially compensated donor centers [6]: 

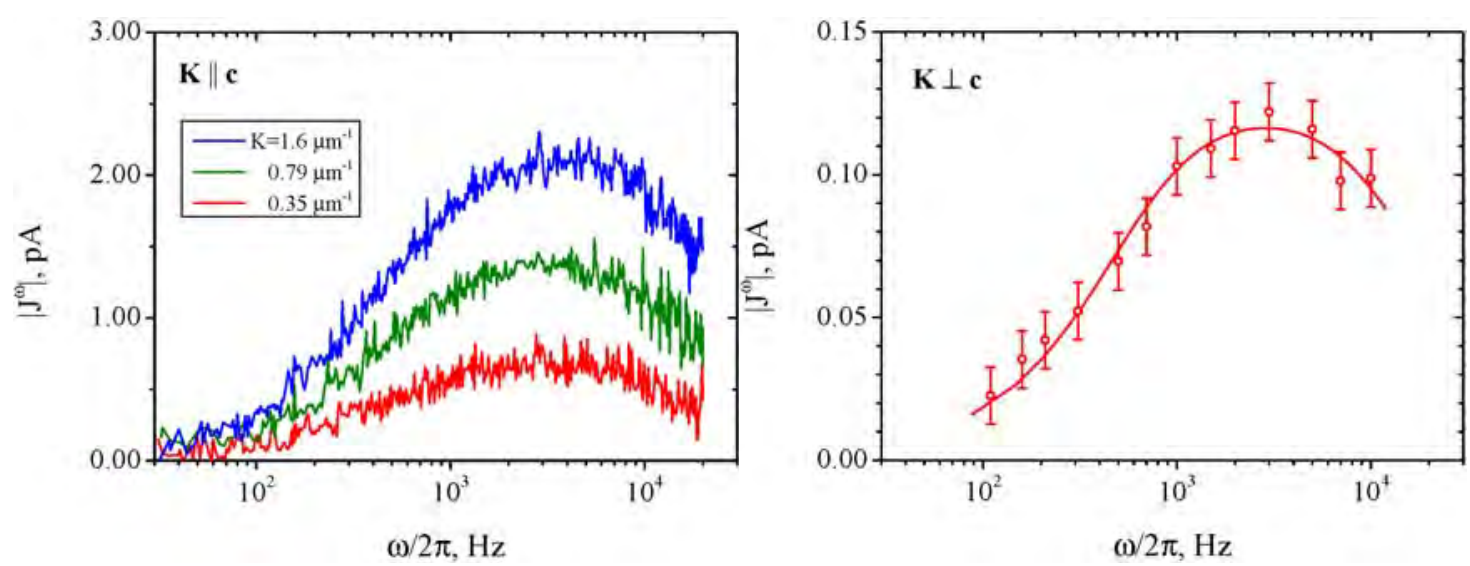

Fig. 2. Frequency transfer functions of the non-steady-state photo-EMF signal in AlN crystal. The dependencies are measured for two geometries: $\boldsymbol{K}$ c-axis, $K=0.35 \mu^{-1}, 0.79 \mu m^{-1}, 1.6 \mu m^{-1}$ (left figure), and $\boldsymbol{K} \perp c$-axis, $K=2 \mu m^{-1}$, (right figure). $I_{0}=110 \mathrm{~mW} / \mathrm{cm}^{2}$. The solid line shows approximation by Eq. (1) with $\omega_{1} / 2 \pi=600 \mathrm{~Hz}$ and $\omega_{2} / 2 \pi=14 \mathrm{kHz}$.

$$
J^{\omega}=J_{m}^{\omega} \frac{i \omega\left(\omega_{2}-\omega_{1}\right)}{\omega_{1} \omega_{2}-\omega^{2}+i \omega\left(\omega_{2}-\omega_{1}\right)}
$$

here $J_{m}^{\omega}$ is the current amplitude at the maximum of frequency dependence. The linear growth for low modulation frequencies $(\omega / 2 \pi<700 \mathrm{~Hz}$ in Fig. 2) is an important manifestation of the adaptive nature of space charge formation in wide-gap semiconductor materials. The signal in this frequency region is small since both the space charge field grating and grating of free carriers (photoconductivity grating) follow the movement of the interference pattern. The spatial shift between the gratings is nearly equal to $\pi / 2$ which results in low signal amplitude. For higher modulation frequencies (frequency independent region) the grating with larger relaxation time can be considered as "frozen in", the periodic spatial shifts increase, and the photo-EMF signal reaches its maximum. For very high modulation frequencies $(\omega / 2 \pi>13 \mathrm{kHz}$ in Fig. 2$)$ the oscillation amplitudes

$$
J^{\omega}(K) \propto \frac{\sigma_{0} E_{D}}{1+\left(K L_{D}\right)^{2}} .
$$

The diffusion length of electrons was estimated from the measured dependence: $L_{D}=0.58 \mu \mathrm{m}$ and $L_{D}=0.92 \mu \mathrm{m}$ for the $\Gamma$ - and $\perp$-geometries, respectively. As seen the signal amplitude decays faster than $K^{-1}$ and changes its sign for high spatial frequencies. A similar effect was observed earlier in GaAs crystal at $\lambda=1.15 \mu \mathrm{m}$ [11]. Such behavior is of both gratings as well as corresponding relative shifts between them become negligible, and the resulting current vanishes.

The dependence of the signal amplitude versus spatial frequency of the interference pattern, i.e. $\mathrm{J}^{\omega}(\mathrm{K})$, is another important characteristic, that is usually measured in the non-steady-state photo-EMF experiments (Fig. 3). The behavior of the signal can be easily explained: the signal increases for low $\mathrm{K}$ due to the growth of space charge field amplitude which is proportional to the so-called diffusion field $\mathrm{E}_{\mathrm{D}}=\mathrm{Kk}_{\mathrm{B}} \mathrm{T} / \mathrm{e}$, the signal decrease for high $\mathrm{K}$ is resulted from the diffusion blurring of the conductivity grating. The dependencies were fitted by the following simple expression known from the non-steady-state photo-EMF theory [6]:

explained by the presence of bipolar photoconductivity: the sign of the signal for low spatial frequencies is determined by the carriers with larger photoconductivity, while the carriers with larger generation rate define the sign of the signal for high $\mathrm{K}$. The expression for photo-EMF amplitude can be written in this case as follows: 
$J^{\omega}(K) \propto \frac{\sigma_{0}^{e} E_{D}}{1+\left(K L_{D}^{e}\right)^{2}}-\frac{\sigma_{0}^{h} E_{D}}{1+\left(K L_{D}^{h}\right)^{2}}$,
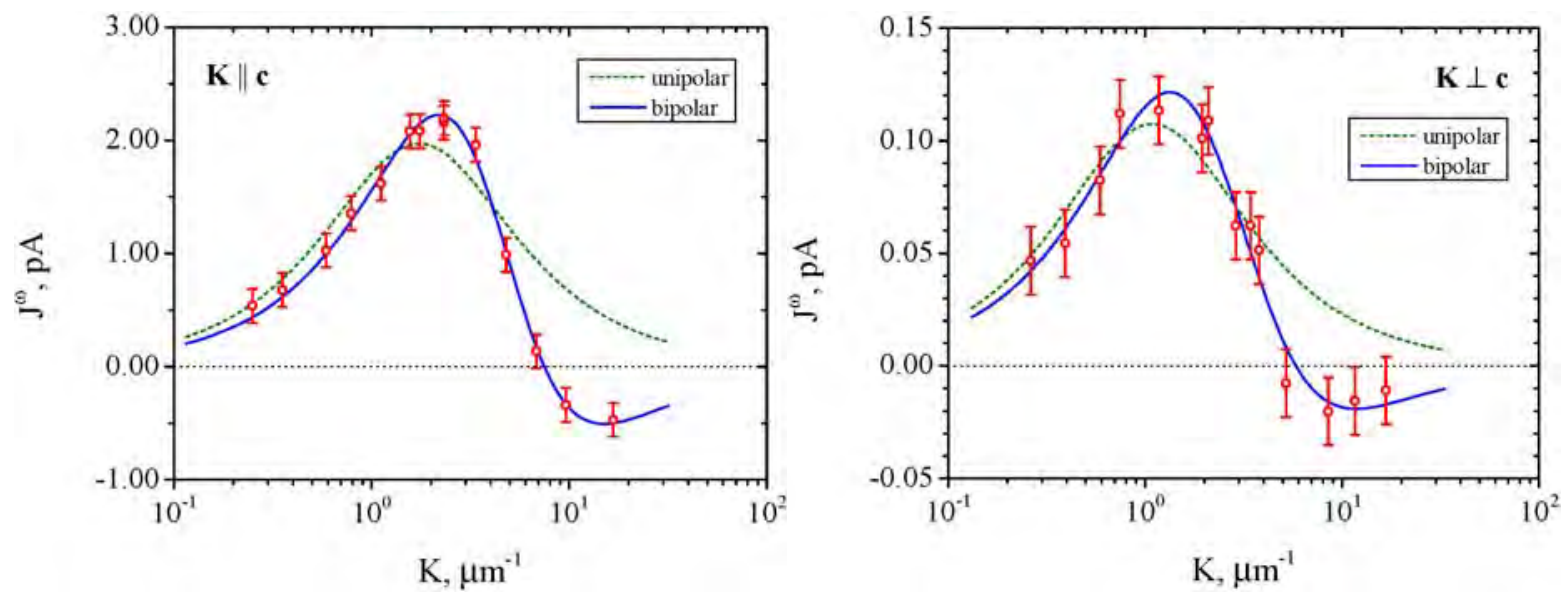

Fig. 3 Dependence of the non-steady-state photo-EMF amplitude on the spatial frequency of the interference pattern. The dependencies are measured for two geometries: $\boldsymbol{K}$ c-axis (left figure), and $\boldsymbol{K} \perp c$-axis (right figure). $I_{0}=110 \mathrm{~mW} / \mathrm{cm}^{2}$. Dotted and solid lines show approximation by Eqs. (2) and (3).

where $\sigma_{0}{ }^{e, h}$ and $L_{D}^{e, h}$ is the average photoconductivities and diffusion lengths of electrons and holes, respectively. One can note that this expression fits experimental dependencies much better (Fig. 3), and it describes the change of the signal sign. The best fit was obtained for the following combination of material parameters: $\sigma_{0}{ }^{\mathrm{e}} / \sigma_{0}{ }^{\mathrm{h}}=1.8, \mathrm{~L}_{\mathrm{D}}{ }^{\mathrm{e}}=0.27 \mu \mathrm{m}, \mathrm{L}_{\mathrm{D}}{ }^{\mathrm{h}}=0.18 \mu \mathrm{m}$ for $\Gamma$ - geometry and $\sigma_{0}{ }^{\mathrm{e}} / \sigma_{0}{ }^{\mathrm{h}}=5.3, \mathrm{~L}_{\mathrm{D}}{ }^{\mathrm{e}}=0.58 \mu \mathrm{m}$, $\mathrm{L}_{D}{ }^{\mathrm{h}}=0.20 \mu \mathrm{m}$ for $\perp$ - geometry. Characteristic Maxwell relaxation time $\tau_{\mathrm{M}}$ and conductivity relaxation time $\tau$ for the $\perp$ - geometry were also estimated from the only transfer function shown in Fig. 2: $\tau_{\mathrm{M}}=0.26 \mathrm{~ms} \tau=12 \mu \mathrm{s}$. The corresponding photoconductivity equals $\sigma_{0}=$ $2.610^{-9} \Omega^{-1} \mathrm{~cm}^{-1}$. Generally speaking, the bipolar conductivity of AIN crystal is unexpected in itself. The energy of photons is of $2.3 \mathrm{eV}$, whereas the band gap of the material is of $6.2 \mathrm{eV}$. This means that electrons and holes can not be generated from the same local levels. There must be at least two groups of levels in the forbidden gap providing extrinsic absorption of the green light and corresponding independent generation of electrons and holes.

\section{Conclusion}

To summarize we studied the effect of non-steadystate photo-EMF in new photosensitive material -aluminium nitride bulk crystal. The significant anisotropy of the material was revealed in the experiments with excitation of the photo-EMF along and across [0001] axis. The presence of the bipolar photoconductivity was established from the change of the signal sign for high spatial frequencies. The average photoconductivity of the material was estimated from the measurements of the signal amplitude versus frequency of phase modulation. The dependence of the signal versus spatial frequency provided the estimation of the diffusion lengths of electrons and holes.

The obtained results have demonstrated that the nonsteady-state photo-EMF technique can be considered as a powerful tool for investigation of wide band gap semiconductors. A number of photoelectric parameters are estimated using the only arrangement. In contrast to the standard methods of semiconductor characterization the technological problem of ohmic contacts for high-resistive materials is not so significant here, since the effect of the non-steady-state photo-EMF is based on the excitation of an alternating current.

\section{Acknowledgements}

The financial support from the Ministry of Knowledge Economy of Korea is gratefully acknowledged.

\section{References}

[1] Y. Taniyasu, M. Kasu, and T.Makimoto, “An aluminium nitride light-emitting diode with a wavelength of 210 nanometres", Nature (London), 441, 325-328 (2006); doi: 10.1038/nature04760.

[2] K. Taylor and C. Lenie, "Some Properties of Aluminum Nitride", J. Electrochem. Soc., 107, 308-314 (1960); doi: 10.1149/1.2427686.

[3] G. Slack, R. Tanzilli, R. Pohl, and J. Vandersande, "The intrinsic thermal conductivity of AIN", J. Phys. Chem. Solids, 48, 641-647 (1987); doi: 10.1016/0022-3697(87)90153-3. 
[4] V. Chin, T. Tansley, and T. Osotchan, "Electron mobilities in gallium, indium, and aluminum nitrides" J. Appl. Phys., 75, 7365 (1994); doi: 10.1063/1.356650.

[5] T. Nagai, R. Fujimura, T. Shimura, and K. Kuroda, "Photorefractive effect in undoped aluminum nitride", Optics Letters 35, 2136-2138 (2010); doi: 10.1364/OL.35.002136.

[6] M. Petrov, I. Sokolov, S. Stepanov, and G. Trofimov, "Non-steady-state photo-electromotive-force induced by dynamic gratings in partially compensated semiconductors" J. Appl. Phys., 68, 2216-2225, (1990); doi: 10.1063/1.346525.

[7] I. Sokolov and S. Stepanov, "Non-steady-state photoelectromotive force in crystals with long photocarrier lifetimes"J. Opt. Soc. Am. B, 10, 1483-1488 (1993); doi: 10.1364/JOSAB.10.001483.

[8] E. Mokhov, O. Avdeev, I. Barash, T. Chemekova, A. Roenkov, A. Segal, A. Wolfson, Y. Makarov, M. Ramm, and H. Helava, "Sublimation growth of AIN bulk crystals in Ta crucibles", J. Cryst. Growth, 281, 93-100 (2005); doi: 10.1016/j.jcrysgro.2005.03.016.

[9] A. Collins, E. Lightowlers, and P. Dean, "Lattice vibration spectra of Aluminum Nitride", Phys. Rev. 158, 833-838 (1967); .doi: 10.1103/PhysRev.158.833.

[10] W. Moore, J. Freitas, R. Holm, O. Kovalenkov, and V. Dmitriev, "Infrared dielectric function of wurtzite aluminum nitride", Appl. Phys. Lett,. 86, 141912-1-3 (2005); doi: 10.1063/1.1899233.

[11] S. Stepanov and G. Trofimov, "Transient EMF in crystals having ambipolar photoconductivity", Sov. Phys. Solid State 31, 49-50 (1989). 